\title{
Mobilisation de l'analyse fonctionnelle pour l'étude de la résilience des quartiers et des systèmes de transport guidé face au risque inondation
}

\section{Mobilization of Functional Analysis for the Resilience Study of Neighbourhoods and Transport Guided Systems against Flood Risk}

\author{
M. Gonzva*,***, M. Balsells**,****, B. Barroca* \\ *Université Paris-Est Marne-la-Vallée, Lab'Urba (ex LEESU) - Département Génie Urbain, 6-8 av. Blaise \\ Pascal - Cité Descartes - Champs sur Marne, 77455 Marne La Vallée CEDEX 2, France \\ (E-mail : bruno.barroca@u-pem.fr) \\ ** Université de Mons, Faculté d'Architecture et d'Urbanisme, Place du Parc 23, 7000, Mons, Belgique \\ (E-mail : mireia.balsells@umons.ac.be) \\ *** SYSTRA, 72, rue Henry Farman, 75015 Paris, France \\ (E-mail : mgonzva@systra.com) \\ **** Université Paris-Est, École des Ingénieurs de la Ville de Paris, 78-80 rue Rébéval, 75019 Paris, France \\ (E-mail : mireia.balsells@eivp-paris.fr)
}

\section{Résumé}

Le risque inondation apparaît aujourd'hui comme l'un des risques naturels les plus catastrophiques car le plus fréquent et dont les effets peuvent être dévastateurs sur le plan environnemental, sociétal et économique. L'expérience de catastrophes passées montre la grande vulnérabilité des systèmes urbains face à ce risque. Or, en tant qu'éléments constitutifs des villes et fondamentaux pour le fonctionnement de celles-ci, leur capacité à absorber une perturbation notamment un aléa naturel est un enjeu majeur futur.

Cet article analyse la résilience face au risque inondation de deux systèmes techniques urbains que sont les systèmes de transport guidé et les quartiers. Les premiers étant physiquement imbriqués dans les seconds, cette imbrication est également fonctionnelle dans la mesure où les systèmes de transport participent au bon fonctionnement des quartiers. Afin de déterminer plus précisément le fonctionnement de ces deux systèmes imbriqués, l'analyse fonctionnelle est mobilisée en tant que méthode issue d'une démarche de sûreté de fonctionnement. L'analyse fonctionnelle permet dans cet article de déterminer de façon formelle et complète la chaîne de dysfonctions générées dans le cas où ces deux systèmes urbains font face à une inondation. Ce travail d'analyse met alors en évidence les éléments urbains les plus vulnérables à cet aléa et les plus critiques au fonctionnement global de la ville.

\footnotetext{
Abstract

Nowadays, the flood risk appears as one of the most catastrophic natural risks it is the most frequent and its impacts can be devastating at the environmental, societal and economic levels. Past disasters revealed the strong vulnerability of urban systems coping flood risk. But, as cities' fundamental components for their functioning, the urban systems' capacity to absorb a disturbance in particular a natural chance is a major stake for the future of our cities.

This paper analyzes the resilience of two urban technical systems in case of flood risk: guided transport systems and neighbourhoods. The first ones are physically imbricated in the second, and this interweaving also exists in a functional aspect insofar as the guided transport systems ensure, amongst others, the proper functioning of neighbourhoods. In order to determine more precisely the functioning of these two imbricated systems, the Functional Analysis is mobilized as a dependability's method for operating safety. In this paper, performing a Functional Analysis brings in a formal and complete way the chain of generated dysfunctions in case the two urban systems studied are facing with flood.
} 
Thus, this analysis highlights the most vulnerable urban systems in case of flood hazard and the most critical ones ensuring the global proper functioning of cities.

\section{Mots-clefs}

Résilience / Analyse fonctionnelle / Inondation / Aménagement des quartiers / Conception des systèmes de transport

\section{Keywords}

Resilience / Functional analysis / Flood / Neighbourhoods design / Transport systems design

\section{Introduction}

La perspective d'un changement climatique combinée à la concentration des biens et des personnes en milieu urbain laisse présager des événements dévastateurs pour les années à venir (Serre et al., 2012). Les changements climatiques semblent de surcroît avoir un rôle amplificateur sur les risques naturels qui doivent alors être réexaminés en fonction de cette nouvelle donne climatique (Quenault, 2013). Parmi ces risques naturels, le risque inondation apparaît comme l'un des plus catastrophiques car le plus fréquent parmi toutes les catastrophes naturelles ${ }^{1}$. Pour illustration, en France, un habitant sur quatre et un emploi sur trois sont en effet potentiellement exposés au risque d'inondation (MEDDE, 2012). Lors d'événements climatiques intenses les systèmes de transport ont montré leur sensibilité au travers d'incidents nombreux (Oslakovic Stipanovic et al., 2013). En outre, les quartiers urbains sont aussi affectés par ces incertitudes et ces risques. Or, leurs nombreuses interactions de dépendances et d'interdépendances avec les autres échelles spatiales urbaines (territoires, villes, etc.) rend encore plus difficile la gestion des risques naturels (Balsells et al., 2013a), en particulier celle du risque inondation. Du côté de la recherche scientifique, depuis le milieu des années 2000, le concept de résilience véhicule une nouvelle manière d'analyser le risque. Dans ce concept, le risque est appliqué à différentes échelles spatiales et temporelles (Serre, 2011) d'une part, et selon une approche systémique d'autre part. La résilience est ainsi vue comme la capacité d'un système à absorber le changement et à persister au-delà d'une perturbation (Barroca et al., 2013). Dans cette communication, nous nous intéressons à l'étude de la résilience urbaine face au risque d'inondation à l'échelle locale du quartier. Cette recherche porte spécifiquement sur la résilience face au risque d'inondation des quartiers et des systèmes de transport, deux systèmes physiquement et fonctionnellement imbriqués. L'enjeu est de déterminer comment le fonctionnement technique de composants urbains structurants tels que les systèmes de transport s'intègre dans le fonctionnement technique des quartiers en contribuant globalement à la résilience des villes face à des risques d'inondation.

\section{Résilience urbaine face au risque d'inondation}

\section{1. Émergence de la résilience dans la gestion des risques d'inondation en milieu urbain}

Compte tenu de l'actuelle situation concernant les catastrophes naturelles et les perturbations sociales qui affectent les systèmes urbains, la résilience urbaine est présentée comme un moyen par lequel les systèmes urbains peuvent faire face à des chocs inattendus et atteindre la durabilité au fil du temps (Toubin et al., 2014). En effet, il apparaît qu'«améliorer la résilience augmente les chances d'un développement durable dans un environnement changeant où le futur est imprévisible et la surprise est

\footnotetext{
${ }^{1}$ EM-DAT : The OFDA/CRED International Disaster Database - www.emdat.be.
} 
probable $»^{2}$ (Folke et al., 2002). Dans ce contexte, la résilience est clairement reconnue comme un nouveau paradigme dans la gestion des risques d'inondation permettant, en définitive, de faire face à des environnements complexes (Lhomme et al., 2013). Ainsi, la perturbation n'est plus uniquement considérée comme un événement négatif mais révèle également des mécanismes inconnus et joue un rôle important dans le fonctionnement, l'évolution et l'adaptation du système urbain. C'est à partir de cette différence que la résilience représente un changement concernant les stratégies traditionnelles de gestion des risques d'inondation qui sont essentiellement basées sur la réduction de la vulnérabilité via des mesures de résistance, de protection. La résilience offre une approche plus globale que la réduction de vulnérabilité en considérant les interactions entre les systèmes urbains et notamment leur emboitement physique et fonctionnel.

\subsection{Opérationnalité de la résilience urbaine face au risque d'inondation}

Bien que la résilience urbaine permette de dépasser conceptuellement et méthodologiquement les analyses sectorielles comme nous l'avons vu, il est difficile de rendre le concept opérationnel car il donne lieu à de multiples traductions en termes de problématique et de développement méthodologique (Toubin et al., 2012). En effet, les actions visant à la mise en œuvre ou à l'évaluation opérationnelle de celle-ci sont encore limitées (Barroca et al., 2012). Néanmoins, concernant la gestion des risques d'inondation en milieu urbain, toujours dans une approche théorique, plusieurs études et projets de recherche visant à rendre opérationnelle la résilience urbaine face au risque d'inondation, peuvent être identifiés (Balsells et al. 2014). En utilisant des approches diverses selon les différentes dimensions urbaines et échelles spatiales concernées, ces travaux ont pour objectif principal de développer des outils (modèles, attributs, indicateurs, etc.) et des méthodes pour analyser et/ou évaluer la résilience urbaine face au risque d'inondation. L'objectif second de ces travaux est d'intégrer ce concept dans les pratiques urbaines actuelles. Il ressort de la tendance suivante : à ce jour, il n'existe pas - ou peu - de références identifiées concernant des études centrées sur l'opérationnalisation de la résilience face au risque d'inondation d'une manière holistique à l'échelle spécifique du quartier.

La méthode de l'Analyse Fonctionnelle (AF) issue de la Sûreté de Fonctionnement est mobilisée, appliquée à chacun de ces deux systèmes urbains. Pour appliquer l'AF, nous montrons dans cette communication qu'il s'avère nécessaire de choisir une granularité pertinente de travail, qui correspond ici à une échelle locale, le quartier. Cette double modélisation fonctionnelle permet de démontrer l'imbrication fonctionnelle des deux systèmes étudiés et offre alors l'opportunité de déterminer leur fonctionnement en situation de crise : lorsqu'une inondation survient. En effet, en situation d'aléa, il est possible, d'une part, d'avoir une meilleure compréhension de la cause exacte du dysfonctionnement du système de transport guidé (le système « imbriqué ») et, d'autre part, une meilleure compréhension des conséquences du dysfonctionnement sur le quartier (le système «imbriquant»). Cette imbrication fonctionnelle est d'autant plus essentielle à mettre en évidence dans la mesure où une défaillance de l'un ou l'autre des systèmes urbains peut entraîner un effet domino difficile à prédire pouvant ainsi engendrer des conséquences néfastes pour la société ou pour l'environnement (Robert \& Morabito, 2009).

L'enjeu est ainsi d'examiner comment l'AF, lorsqu'elle est appliquée jusqu'à une certaine échelle urbaine et à certains systèmes urbains physiquement imbriqués, peut-elle apporter une meilleure compréhension de la résilience face au risque inondation des quartiers, et donc des villes.

\section{Méthodes de Sûreté de Fonctionnement : l'Analyse Fonctionnelle}

\subsection{Analyse Fonctionnelle et applications en génie urbain}

\footnotetext{
2 « Managing for resilience enhances the likelihood of sustaining development in changing environments where the future is unpredictable and surprise is likely ».
} 
Les méthodes de la Sûreté de Fonctionnement ont été développées à l'origine pour étudier des systèmes industriels au fonctionnement complexe (Villemeur, 1998). Or, depuis un certain nombre d'années, des travaux de recherche appliquée mobilisent ces méthodes pour la modélisation du fonctionnement de systèmes complexes de type urbain. Parmi ces méthodes existe l'Analyse Fonctionnelle qui est une des plus utilisées en Sûreté de Fonctionnement. Elle permet de modéliser le fonctionnement de systèmes complexes à partir de deux analyses dépendantes l'une de l'autre: une analyse structurelle et une analyse fonctionnelle. L'analyse structurelle permet de définir les relations des différents composants du système pour formuler, dans l'analyse fonctionnelle, les fonctions de chaque composant. Deux outils sont alors mis en œuvre pour représenter le système : le Bloc-diagramme Fonctionnel (BdF) et le Tableau d'Analyse Fonctionnelle (TAF) (Zwingelstein, 1996).

Cette méthode a été appliquée dans plusieurs travaux de génie urbain (Fig.1) car elle est un outil préalable puissant pour déterminer le modèle de fonctionnement, et donc de dysfonctionnement, de systèmes complexes lorsqu'ils font face à une crise. En effet, préalable car, l'AF peut être notamment associée à l'Analyse des Modes de Défaillance et de leurs Effets (AMDE). L'AF permet ainsi d'étudier la résilience de systèmes tels que les digues (Serre, 2005; Vuillet, 2012), les réseaux techniques urbains (Lhomme, 2012) ou encore le service de gestion des déchets (Béraud, 2013) face à l'aléa naturel d'inondation. D'autres applications en génie urbain existent, non nécessairement associées à la résilience urbaine. Citons les travaux de (Peyras, 2009) sur les barrages, de (Allaire, 2012) sur les performances énergétiques de bâtiment, de (Correc, 2005) sur les installations d'eau à l'intérieur des bâtiments, de (Alard et al., 2013) sur le traitement des eaux ou encore de (Valadas, 2003) sur les routes.

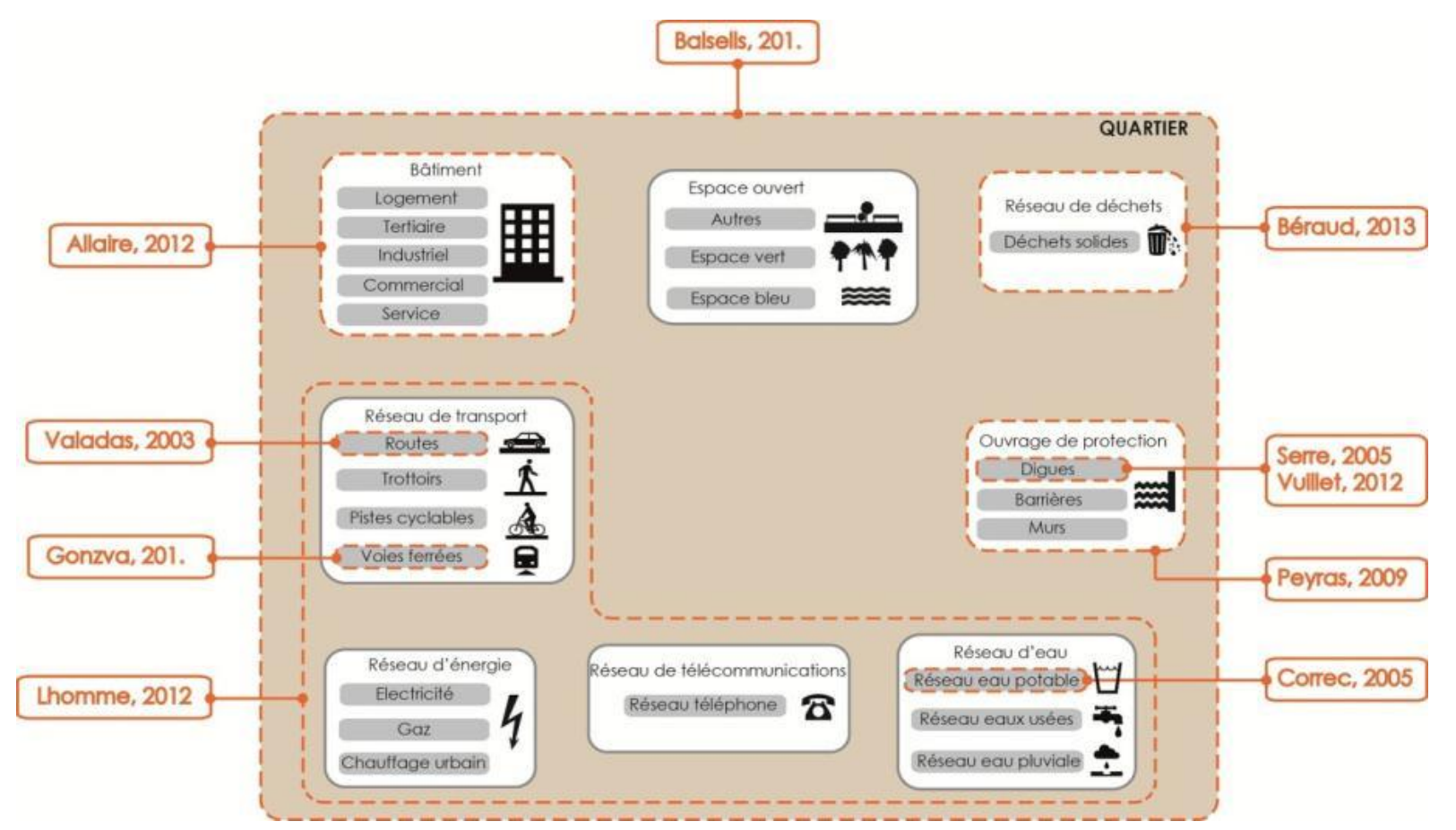

Fig.1. Références d'application de l'Analyse Fonctionnelle en génie urbain

\subsection{Applications de l'Analyse Fonctionnelle aux systèmes étudiés}

\subsubsection{Analyse Fonctionnelle d'un quartier}

Un quartier est une fraction du territoire d'une ville, dotée d'une physionomie propre et caractérisée par des traits distinctifs lui conférant une certaine unité et une individualité (Merlin \& Choay, 2000). Lorsque des fonctions spécifiques sont représentées dans un quartier, des activités, des flux et par conséquent des dynamiques urbaines peuvent être identifiées. Ce travail de modélisation des systèmes urbains, déjà assez bien couvert à l'échelle de la ville (De Rosnay, 1975 ; Cambien, 2008 ; Lhomme, 
2012), apparaît aussi possible à l'échelle du quartier. En effet, un quartier peut être considéré comme un système ouvert, complexe, lequel est caractérisé par des processus d'échange avec son environnement, et en constante évolution et développement.

L'AF d'un quartier correspond ici précisément à l'analyse des fonctions d'ordre technique assurées par les systèmes d'un quartier, par opposition à des fonctions d'ordre plutôt sociétal (se loger, se nourrir ou travailler) qu'assurent aussi un quartier. Pour réaliser l'AF d'un quartier, il est nécessaire d'abord de fixer le niveau de granularité spatiale adapté aux besoins de l'étude. Qualitativement, trois niveaux urbains peuvent être identifiés: un niveau macro (le système urbain étudié), un niveau méso (les composants du système étudié) et enfin un niveau micro (les sous-composants et matériaux des composants du niveau macro). Pour effectuer la description détaillée d'un quartier et de son fonctionnement, le rang intermédiaire méso paraît adapté et permet de focaliser l'analyse sur les fonctions assurées par chaque composant d'un quartier.

Ensuite, l'analyse structurelle du quartier précise et liste les composants du quartier pour déterminer, durant l'analyse fonctionnelle, les interactions avec les autres composants. Ces interactions interne entre les composants et externe avec l'environnement sont représentées au moyen d'un BdF (Fig.2).

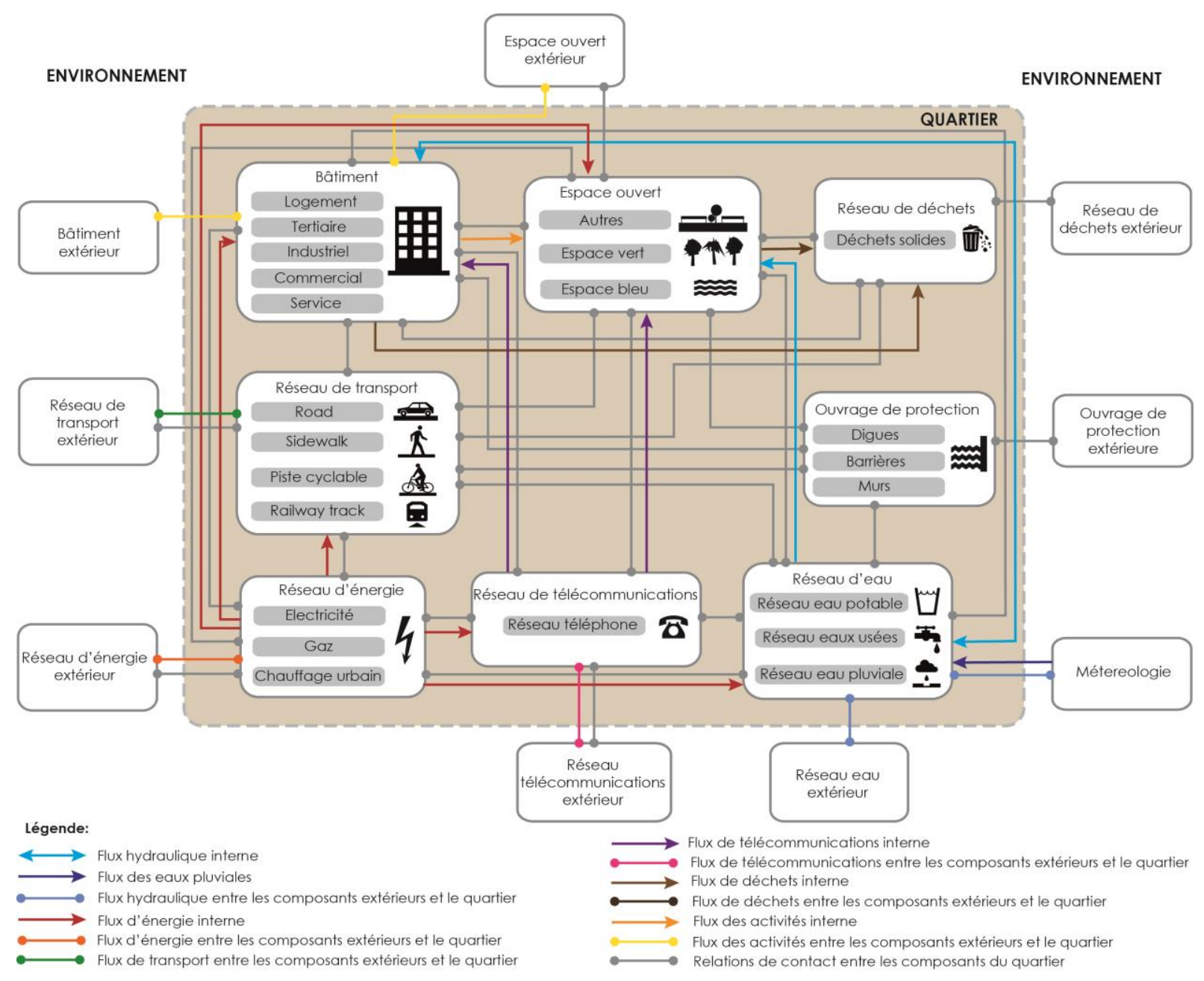

Fig.2. Bloc-diagramme Fonctionnel du système quartier (Balsells et al., 2013b)

\subsubsection{Analyse Fonctionnelle d'un système de transport}

Le second système urbain qui fait l'objet d'une AF est le système de transport guidé. Les systèmes de transport guidé urbains concernent (CERTU, 2007) :

- les métros, en site propre intégral et généralement souterrains, en conduite manuelle, automatique ou automatique légère ; 
- les tramways, caractérisés par des véhicules ferroviaires (roulement fer sur fer) qui circulent majoritairement sur la voirie urbaine et exploités en conduite à vue ;

- les systèmes guidés sur pneus, caractérisés par un roulement sur pneus et un guidage par rail central, par caméra ou magnétique.

Dans un premier temps, l'analyse structurelle permet de déterminer les composants du système et les éléments de son environnement. Ces derniers sont établis à partir des Spécifications Techniques d'Interopérabilité (STI), directives européennes relatives à l'interopérabilité du système ferroviaire européen. Dans cette optique, les STI formulent un découpage du système ferroviaire qui sert de base à la décomposition du système étudié, complétée par d'autres composants considérés comme nécessaires au fonctionnement d'un système de transport guidé. Le système se divise ainsi en huit composants (Gonzva et al., 2014) :

- Contrôle-commande et signalisation : permet de garantir la circulation des trains en toute sécurité ;

- Énergie : correspond à la fourniture en énergie de traction du matériel roulant ;

- Gare ;

- Infrastructure : correspond aux équipements fixes relatifs aux voies ;

- Matériel Roulant;

- Poste de Commandement Centralisé (P.C.C.) : assure l'exploitation par une gestion en temps réel du système de transport ;

- Site de Maintenance des Infrastructures (S.M.I.) : assure la maintenance des équipements fixes relatifs aux voies, à l'énergie et au contrôle-commande et signalisation ;

- Site de Maintenance et de Remisage (S.M.R.) : assure la maintenance en atelier du matériel roulant (dépannage, entretien, remplacement) et son remisage en période hors-exploitation.

De plus, l'environnement du système de compose de :

- Usagers : utilisent le système de transport guidé dans leurs déplacements ;

- Sous-stations électriques : assurent l'alimentation en énergie électrique d'une section de cette ligne.

Dans un second temps, sont étudiées les relations de contacts qui existent entre les composants du système eux-mêmes et entre les composants du système et les éléments de l'environnement. Elles traduisent l'existence d'au moins une connexion physique, matériel entre les deux éléments (matériel roulant alimenté par l'énergie, circulant sur l'infrastructure, etc.). Ces relations de contact permettent au système d'assurer le service de transport des usagers à partir des fonctions qu'assure chaque composant. Un second temps d'analyse fait apparaître les flux de dépendances entre tous ces éléments. En effet, l'existence d'un flux orienté entre deux composants ne peut se réaliser que dans la mesure où le bon fonctionnement du premier composant est assuré et que le lien entre ces deux composants a la capacité à supporter le flux.

Le BdF des relations de contact et flux de dépendances en situation normale est présenté (Fig.3). Ce BdF permet de mettre en évidence un certain nombre de relations de dépendances, faibles, moyennes ou fortes. 


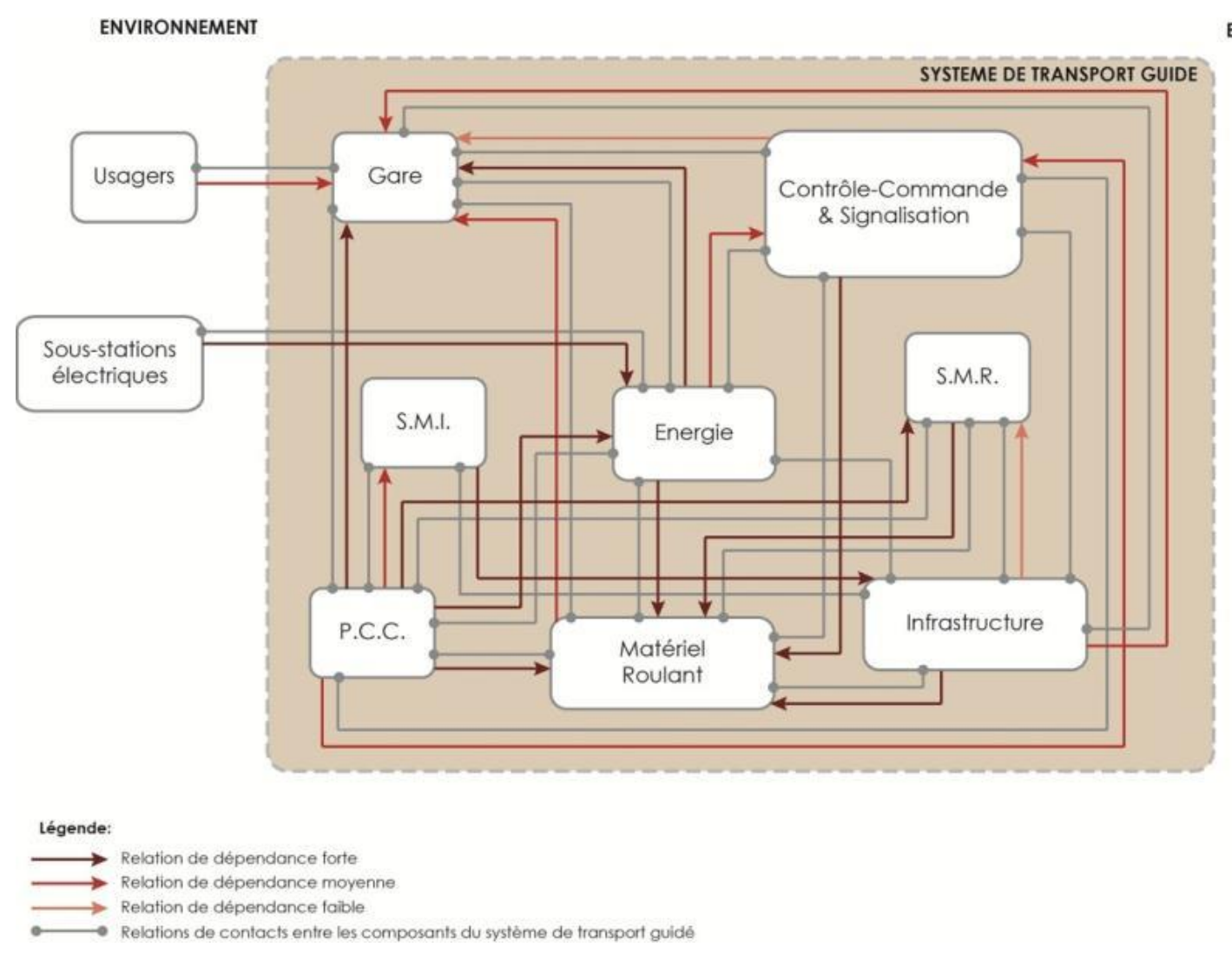

Fig.3. Bloc-diagramme Fonctionnel du système de transport guidé

\section{Discussion}

\subsection{Imbrication locale des analyses fonctionnelles}

Le système de transport guidé est un composant technique du système quartier dans la mesure où, dans les faits, le premier est physiquement inclus dans le second. L'imbrication des analyses fonctionnelles de ces systèmes apporte une meilleure compréhension du fonctionnement général du système englobant, par rapport au système englobé. En particulier, dans le cas où le système quartier fait face à une inondation, l'imbrication des analyses fonctionnelles semble pouvoir déterminer de façon précise et complète la chaîne de dysfonctions générées (Fig.4). 


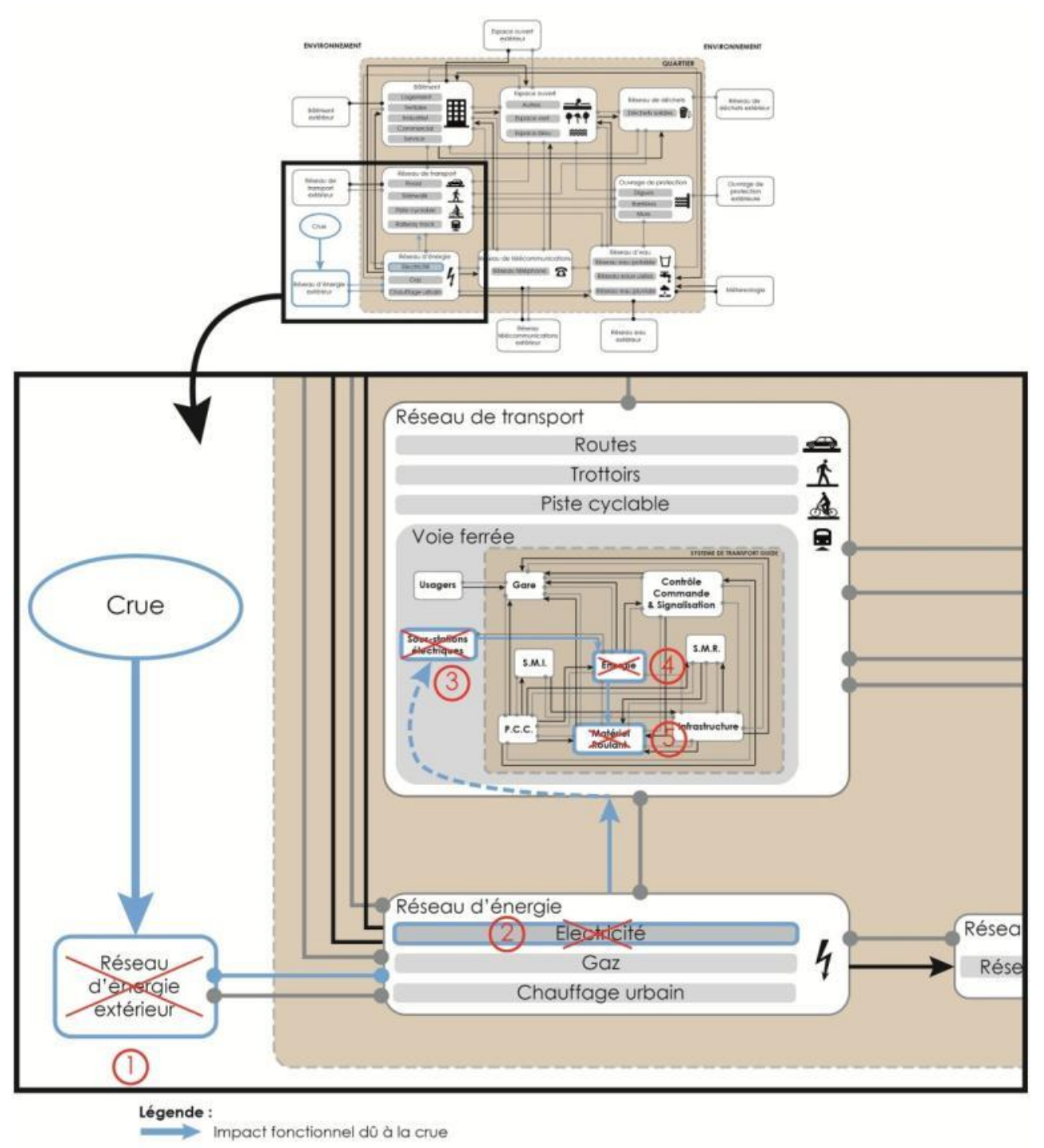

Fig.4. Imbrication des analyses fonctionnelles du quartier et du système de transport guidé

Pour illustrer cette imbrication fonctionnelle, si une inondation touche (1) le «réseau d'énergie extérieur » du quartier alors les composants présentant un flux d'échange avec le « réseau d'énergie » du quartier risque de mettre en dysfonctionnement ce dernier. Ce faisant, la partie (2) «électricité » du " réseau d'énergie » risque à son tour de dysfonctionner. Le « flux d'énergie interne » reliant le « réseau d'énergie » du quartier au « réseau de transport » local implique donc une perturbation du réseau de transport du quartier. A cette étape l'imbrication de l'AF du système de transport guidé avec celle du quartier révèle les dysfonctionnements au sein des éléments du composant " réseau de transport » émanant de sa dépendance fonctionnelle au quartier. L'interruption du service électrique qui implique une dysfonction (3) des «sous-stations électriques », donc de la partie (4) «Énergie » de traction du système de transport et (5) du « Matériel Roulant » engendre finalement une perturbation du service de transport d'usagers de gares en gares.

Cette illustration d'un cas relativement simple d'inondation affectant initialement le réseau d'énergie du quartier met en évidence la chaîne complète de composants qui dysfonctionnent pour entraîner l'arrêt du service de transport. L'imbrication des analyses fonctionnelles apporte, d'une part, une meilleure compréhension de la cause exacte du dysfonctionnement du système de transport guidé (le système «imbriqué ») et, d'autre part, une meilleure compréhension des conséquences du dysfonctionnement sur le quartier (le système «imbriquant»). La notion de système imbriqué et de 
système imbriquant apporte une meilleure compréhension du caractère résilient (ou non) d'un système à partir du caractère résilient (ou non) de ses composants.

\subsection{Application opérationnelle : exemple de la crue de la Seine de 1910}

Pour illustrer cette notion d'imbrication fonctionnelle, le cas de la crue de la Seine de 1910 et de son impact sur le métro parisien de l'époque apparaît pertinent.

La Compagnie du chemin de fer métropolitain de Paris ouvre en 1901 sa propre usine électrique (dite «de Bercy») au 46-48 quai de la Râpée pour produire du courant et alimenter les sous-stations électriques de la nouvelle ligne 1 . Elle dispose de chaudières produisant de la vapeur qui est transformée en électricité par des alternateurs. Ce courant haute tension ainsi produit sert donc pour l'alimentation en courant de traction et d'éclairage de la ligne (Robert, 1967). Or, dès les premiers jours de la crue de la Seine, en 1910, le quartier de Bercy est envahi par les eaux ce qui conduit à l'inondation progressive de l'usine de Bercy à partir du 21 janvier. Ainsi, dès le 22 janvier au matin, l'exploitation de la ligne 1 est interrompue faute de fourniture en énergie de traction électrique. Elle sera rétablie de façon sporadique les jours suivants grâce à l'utilisation du courant électrique d'une autre usine non atteinte par les eaux (Hétier \& Bienvenüe, 1910).

Cette succession d'évènements qui a conduit à l'interruption de service de la ligne 1 se décrit parfaitement au sein de l'imbrication fonctionnelle. En effet, en reprenant la chaîne de dysfonctionnements des composants du paragraphe précédent, il est possible de l'appliquer à la crue de 1910 : la « crue » atteint l'usine électrique de Bercy (1) perturbant la production de courant électrique (2), ce qui entraîne la défaillance des sous-stations électriques (3) de la ligne de métro, qui n'est alors plus alimentée en énergie de traction (4), interrompant finalement la circulation des véhicules ferroviaires (5).

Les enjeux actuels par rapport à la situation de 1910 sont devenus plus nombreux. Actuellement en Îlede-France, la région présente un grand nombre d'équipements ferroviaires stratégiques pour l'exploitation du réseau qui sont situés en zone inondable. La sous-station électrique Pompadour qui alimente en électricité les voies du RER D de Maisons-Alfort Alfortville à Villeneuve Saint-Georges et la zone de Triage de Villeneuve Saint-Georges est un exemple d'équipements fortement soumis à l'aléa inondation. Une crue centennale inonderait en effet la sous-station sous deux mètres d'eau (Mathieu, 2007). Cependant, RTE (Réseau de Transport d'Electricité) s'est engagé à assurer à tout moment de la crue l'alimentation électrique d'autres sous-stations situées le long du RER D de sorte qu'en cas de submersion de la sous-station Pompadour, la puissance perdue serait en partie reprise par les autres sous-stations pour garantir la circulation des voies encore exploitables. Ces mesures organisationnelles devraient permettre d'éviter une situation telle que celle de l'usine de Bercy en 1910.

\subsection{Quelle imbrication des analyses fonctionnelles à une échelle plus large ?}

Comme montré précédemment, l'échelle urbaine locale, représentée par le quartier, apparaît opérationnelle pour mobiliser l'imbrication des analyses fonctionnelles dans l'étude de la résilience face au risque inondation.

À des échelles plus larges de plusieurs quartiers connectés entre eux par des systèmes de transport guidé, la complexité du système semble devient limitante pour l'imbrication fonctionnelle. L'étude ne porte alors plus sur un système (le quartier) mais plutôt sur un ensemble de systèmes (plusieurs quartiers) complexes. Dans ce cas, les interactions entre la multitude de composants étudiés sont teintées de nombreuses interdépendances: interactions multiples, redondances fonctionnelles (notamment au niveau des réseaux d'énergie). Dans l'illustration précédente (Fig.6), le fait que la crue atteigne (1) le «réseau d'énergie extérieur » ne conduit pas nécessairement à la dysfonction (2) du « réseau d'énergie » électrique dans la mesure où, à l'échelle de plusieurs quartiers, ce réseau peut avoir un maillage avec redondance qui permet la continuité fonctionnelle. L'imbrication des systèmes complexifie grandement l'étude car le niveau d'imbrication est généralement très important. La mise en 
œuvre subséquente d'autres méthodes peuvent alors être nécessaires afin de dépasser cette complexité. Cette réflexion rejoint une des hypothèses formulées par (Lhomme, 2012) selon laquelle il n'est possible de modéliser des scénarios de défaillances de systèmes de systèmes complexes qu'en utilisant une combinaison de méthodes issues de la Sûreté de Fonctionnement. 


\section{Conclusion}

Cette communication porte sur l'étude de la résilience face au risque inondation des quartiers et des systèmes de transport guidé. Ces deux systèmes urbains sont imbriqués physiquement, cette imbrication est étudiée à l'aide de la méthode de l'Analyse Fonctionnelle issue de la Sûreté de Fonctionnement. En appliquant cette méthode successivement aux deux systèmes, nous démontrons l'imbrication fonctionnelle qui existe dans leurs interactions, en situation normale. Cette modélisation fonctionnelle donne l'opportunité de déterminer leur fonctionnement technique en situation de crise et ainsi prépare la mise en place d'action pour favoriser la continuité de service. Plusieurs conclusions peuvent être tirées de l'approche méthodologique :

- il est nécessaire de fixer une unique échelle d'étude, l'AF ne semble pas permettre les analyses inter scalaires, trop compliquées à mettre en œuvre. Or, la précision nécessaire de l'AF pour envisager la continuité des systèmes de transport guidé face à l'inondation impose une étude locale. Il apparaît donc que la mobilisation de l'AF dans la compréhension des conséquences d'un risque naturel ne s'avère pas efficace au-delà d'une échelle urbaine locale ;

- l'AF permet une meilleure compréhension de la cause exacte du dysfonctionnement du système de transport guidé (le système « imbriqué ») ;

- l'AF permet une meilleure compréhension des conséquences du dysfonctionnement sur le quartier (le système « imbriquant ») ;

Pour des risques hydrologiques appliqués à des systèmes de transport guidé, la granularité de l'étude ne semble, pas pouvoir s'étendre à une échelle urbaine autre que locale. Cette limite est issue de l'approche systémique elle-même qui, au-delà de l'échelle urbaine locale, ne considère plus des systèmes complexes mais des systèmes de systèmes complexes. Or dans ce cas, l'AF, à elle seule du moins, ne permet pas de modéliser des scénarios de défaillances en situation d'aléa. Les imbrications mettent en exergue les composants touchés par les effets domino générés par une inondation, sans quantifier la probabilité de réalisation de ces chaînes de défaillances successives. Quoi qu'il en soit, l'analyse fonctionnelle apparaît comme un outil pertinent pour analyser la résilience de systèmes urbains face au risque d'inondation, en mettant en évidence les composants les plus vulnérables à cet aléa et les plus critiques au fonctionnement global du système. Ainsi, ce travail apporte dans une certaine mesure une aide pour la définition d'actions et/ou de stratégies d'amélioration de la résilience à des échelles urbaines locales en ciblant les composants. 


\section{Références}

Alard S., Hangouet J.-P., Guy B. (2013). « La sûreté de fonctionnement : démarche pour maîtriser les risques dans les activités du traitement de l'eau ». Revue TSM, Nº10, Octobre 2013, pp. 19-26.

Allaire D. (2012). Développement d'une approche systémique de la gestion patrimoniale d'un parc immobilier d'envergure nationale pour améliorer sa performance énergétique - Une application réalisée sur le parc immobilier de l'État utilisé par le ministère de la défense. Thèse de doctorat. Université Paris-Est.

Balsells M., Barroca B., Diab Y., Becue V., Serre D. (2013a). Améliorer la résilience urbaine face au risque d'inondation : proposition d'une méthodologie, Colloque futurs urbains, Enjeux interdisciplinaires émergents pour comprendre, projeter et fabriquer la ville de demain, Université de Paris-Est, Marne-La-Vallée, 16-18 janvier 2013

Balsells M., Barroca B., Diab Y., Becue V., Serre D. (2013b). Urban design contribution to neighbourhood flood resilience: proposition of a model analysis, International Conference on Flood Resilience: Experiences in Asia and Europe, 5-7 September 2013, Exeter, United Kingdom.

Balsells M., Barroca B., Becue V., Serre D. (2014). Making urban flood resilience more operation: a review of current practices, Water Management journal (à paraître).

Barroca B., Serre D., Diab Y. (2012). « Le concept de résilience à l'épreuve du génie urbain ».

Barroca B., Di Nardo M., Mboumoua I. (2013). «De la vulnérabilité à la résilience : mutation ou bouleversement? ». EchoGéo, $\mathrm{N}^{\circ} 24$.

Béraud H. (2013). Initier la résilience du service de gestion des déchets aux catastrophes naturelles - Le cas des territoires urbains et de l'inondation. Thèse de doctorat. Université Paris-Est.

Cambien A. (2008). «Urban Modelling: emergence of a systemic approach of urban development », $7^{\text {th }}$ Congress of the UES Systems Science European Union Lisbon, 17-19 Dec. 2008.

CERTU \& CETE de Lyon, 2007, Panorama des villes à transports publics guidés. 53 p.

Correc 0. (2005). Approche possibiliste de prédiction des risques de dégradation des réseaux de distribution d'eau à l'intérieur des bâtiments. Thèse de doctorat. Université Marne-la-Vallée.

De Rosnay J. (1975). Le macroscope. Vers une vision globale, Paris, Ed. Seuil.

Folke C., Carpenter S., Elmqvist T., Gunderson L., Holling C. S., Walker B. (2002). « Resilience and sustainable development: building adaptive capacity in a world of transformations ». Ambio, 31, 5, pp. 437-440.

Gonzva, M., Gautier, P.-E., Diab, Y., Barroca, B. (2014). « Résilience des systèmes de transport guidé face aux risques naturels ». Décider dans un monde incertain : enjeu majeur de la maîtrise des risques, Congrès Lambda Mu 19, Dijon, France, 21-23 oct. (à paraître).

Hétier M. \& Bienvenüe F. (1910). Commission Des Inondations - Le Chemin De Fer Métropolitain De Paris, Rapport, 9 avril.

Lhomme S. (2012). Les réseaux techniques comme vecteur de propagation des risques en milieu urbainUne contribution théorique et pratique à l'analyse de la résilience urbaine. Thèse de doctorat, Université Paris-Diderot, 365 p.

Lhomme S., Laganier R., Diab Y., Serre D. (2013). «La résilience de la ville de Dublin aux inondations : de la théorie à la pratique ». Cybergeo : European Journal of Geography.

Mathieu S. (2007). Prévention du Risque Inondation à la SNCF. Mémoire de Master II «Génie Urbain », Université de Marne-la-Vallée.

Merlin P., Choay F. (dir.) (2000). Dictionnaire de l'urbanisme et de l'aménagement, Presses universitaires de France, Paris, 903 p.

Ministère de l'Écologie, du Développement Durable et de l'Énergie (2012). Première évaluation nationale des risques d'inondation, Principaux résultats - EPRI 2011. 
Oslakovic Stipanovic I., ter Maat H., Hartmann A., Dewulf G. (2013). Risk Assessment Of Climate Change Impacts On Railway Infrastructure. In : P. Carrillo \& P. Chinowsky (Eds.), Engineering Project Organization Conference. Devil's Thumb Ranch, Colorado.

Peyras L. (2009). Évaluation de la performance et des risques des ouvrages hydrauliques de génie civil, Mémoire d'HDR, Université Blaise Pascal-Clermont II.

Quenault B. (2013). «Retour critique sur la mobilisation du concept de résilience en lien avec l’adaptation des systèmes urbains au changement climatique ». EchoGéo, N²4, pp.1-19.

Robert J. (1967). Notre Métro, Paris.

Robert B., Morabito L. (2009). Réduire la vulnérabilité des infrastructures essentielles : Guide méthodologique. Éditions Tec\&Doc, 62 p.

Serre D. (2005). Évaluation de la performance des digues de protection contre les inondations Modélisation de critères de décision dans un Système d'Information Géographique. Thèse de doctorat. Université Marne-la-Vallée.

Serre D., Lhomme S., Peyras L., Laganier R., Diab Y. (2012). " Analyzing the civil engineering infrastructures to prioritize urban flood resilient actions ». 7th International Conference on Water Sensitive Urban Design, 21-23 February 2012, Melbourne, Australia.

Toubin M., Lhomme S., Diab Y., Serre D., Laganier R. (2012). « La Résilience urbaine : un nouveau concept opérationnel vecteur de durabilité urbaine ? », Développement durable et territoires, Vol. 3, $\mathrm{N}^{\circ} 1$.

Toubin M., Laganier R., Diab Y., Serre D. (2014). « Improving the conditions for urban resilience through collaborative learning of Parisian urban services ». Journal of urban planning and development, in press.

Valadas E. (2003). Identification des scénarios de dégradations des chaussées bitumineuses épaisses, Mémoire présenté pour l'obtention du diplôme d'ingénieur en génie civil, CUST, Université Blaise Pascal-Clermont II, 148 p.

Villemeur A. (1998). Sûreté de fonctionnement des systèmes industriels. Fiabilité, facteurs humains, informatisation, Collection de la Direction des Études et Recherches d'Électricité de France. Éd. Eyrolles, Paris.

Vuillet M. (2012). Élaboration d'un modèle d'aide à la décision basé sur une approche probabiliste pour l'évaluation de la performance des digues fluviales. Thèse de doctorat. Université Paris-Est Marne-laVallée.

Zwingelstein G. (1996). La maintenance basée sur la fiabilité, Collection Diagnostic et Maintenance, Hermès. 